\title{
The influence of vision on the absolute identification of sound-source position
}

\author{
B. R. SHELTON and C. L. SEARLE \\ Queen's University, Kingston, Ontario, Canada
}

\begin{abstract}
The influence of vision on auditory localization was assessed in an absolute identification paradigm using sighted and blindfolded subjects. Vision improved the accuracy of judgments directly in front of, to the side of, and behind the head of subjects in the horizontal plane, but had little relevance to vertical-plane localization. The exact form of the observed facilitation depended on the orientation of the speaker array to the head. In a second experiment involving sound localization in 10 visual environments, there was evidence for the operation of two distinct influences of vision on directional hearing. One result supported the hypothesis that vision provides a frame of reference for judgments, and a second indicated the importance of vision to the maintenance of spatial memory.
\end{abstract}

Previous research has indicated the importance of vision in the accuracy of auditory localization judgments (Jackson, 1953; Jones, 1975a; Wallach, 1940). The spatial coordination of these two senses has been shown in infants by both the visual orientation of newborns toward a sound source (Muir \& Field, 1979; Wertheimer, 1961) and the sensitivity of slightly older babies to a discrepancy between the locus of the acoustic and visual image of an object (Aronson \& Rosenbloom, 1971; Spelke, 1976). The latter strategy has also proved to be useful with adults, where the interaction between modalities has been demonstrated by an aftereffect of the presentation of discordant visual and auditory spatial information (Canon, 1970; Jack \& Thurlow, 1973; Radeau \& Bertelson, 1974; Thurlow \& Jack, 1973a, 1973b).

With respect to the accuracy of auditory localization, the general finding has been that performance is more accurate in the presence of vision (Annis, 1974; Fisher, 1964; Jackson, 1953; Jones, 1975b; Warren, 1970). Two main hypotheses have been presented to account for the facilitatory effects of sight. The visual frame-of-reference hypothesis has been defended by Warren (1970), whereas Jones (1975a) has argued that the majority of the effect can be accounted for by the visual facilitation of spatial memory.

Warren (1970) reported a number of experiments to support the claim that vision provided a context in which auditory localization judgments were made. In one, subjects localized repetitive clicks in total

B. R. Shetton's present address is: Laboratory of Psychophysics, William James Hall, Harvard University, Cambridge, Massachusetts 02138 . C. L. Searle's present address is: Research Laboratory of Electronics, Building 36-789, Massachusetts Institute of Technology, Cambridge, Massachusetts 02139. darkness, with a single visual marker directly in front, or while touching the light in the dark. Facilitation was found only in the case in which the single light was provided. Since only one visual stimulus was required, and since proprioception could not provide the same facilitation, Warren argued that vision served to organize auditory space. The same author demonstrated that the facilitation was not due to an enhancement of pointing accuracy in that facilitation still held in a same-different paradigm.

McLaughlin and Bower (1965) had subjects judge the position of visual and auditory targets, as well as the position of straight-ahead, as the course of prism adaptation progressed. They reported that errors in auditory localization paralleled those of pointing straight ahead, whereas those of visual direction followed a different time course. In more extensive experiments, Lackner (1973a, 1973b) found that the change in the position of a lateralized image follows the same temporal pattern as the perceived change in the position of the head with respect to the trunk during adaptation to prism displacement. Lackner therefore argued that the disruption of lateralization was related to an apparent change in the frame of reference, a view compatible with Warren's account of visual facilitation of auditory localization.

In some situations, however, target-directed eye movements are required to maximize visual facilitation. Jones and Kabanoff (1975) report that the absolute identification of sound-source position was significantly improved when subjects were allowed to move their eyes. Furthermore, when subjects were presented with a single light to mark the same two speakers, there was an interaction between the spatial concurrence of light and sound, and the freedom of subjects to move their eyes, in the determination of 
the accuracy of performance. Specifically, free eye movements improved performance with concordant visual and auditory stimuli but impaired performance with spatially discordant light and sound. Jones and Kabanoff argued that the interaction was not explicable by a frame-of-reference hypothesis and that the data indicate the importance of eye movements to visual facilitation.

The same interpretation was placed on Jones and Kabanoff's demonstration that the instruction to subjects to move their eyes in the direction of a sound presented in darkness produced better performance than when the eyes remained fixed, but an instruction to move the eyes away from a source degraded auditory localization. The specificity of the ocularmotor components required for facilitation in these experiments led Jones (1975a) to conclude that visual facilitation involved a mechanism similar to the reverbatory circuits described by Sherrington (1947), and that the process involved the facilitation of memory for spatial position. In a review of the effects of blindness on space perception, Jones (1975a) made a case for the hypothesis that blind subjects are debilitated in auditory tasks that require the retention of the spatial position of the stimulus.

These hypotheses have been treated as mutually exclusive in the literature, but it is interesting to note an experiment conducted by Thurlow and Kerr (1970) in which subjects were placed in a revolving striped drum and asked to judge the position of straight-ahead and the position of an unseen sound source. The apparent straight-ahead was shifted in the direction of the drum rotation, as was the position of the sound, but the sound-source position was consistently judged to be displaced farther than the apparent straight-ahead. The additional displacement was in the direction of induced eye movements, and Thurlow and Kerr suggested that there were two components to the bias they observed-one due to a shift of the frame of reference and another related to the visually induced eye movements.

The purpose of the first experiment was to document the relative potency of visual information in the facilitation of absolute-identification judgments. Performance was assessed in three horizontally arrayed spans and in one vertically presented set of speakers. Half the subjects were tested with the benefit of vision, and half were blindfolded during the experiment.

\section{EXPERIMENT 1}

\section{Method and Procedure}

Eighty female undergraduate volunteers with apparently normal hearing localized single 200 -msec bursts of white noise presented at $72 \mathrm{~dB}$ SPL. Eight $11.5-\mathrm{cm}$ speakers were fixed on a 2.25 -m-diam hoop, with a spacing of $11 \mathrm{deg}$. The subject's chair, with headrest and bite plate, was situated such that the subject's head was in the approximate center of the speaker ring. The subjects were provided with a response box, with eight buttons arranged similarly to the speakers. The intertrial interval (ITI) was $7 \mathrm{sec}$.

The subjects were positioned with respect to the horizontally arrayed speaker set so as to orient the array directly in front, directly behind, or along the left side of the head. Specifically, the three horizontal arrays comprised speaker placements at approximately: $\pm 5.5, \pm 17, \pm 28$, and \pm 39 deg azimuth; 0,349 , $338,327,316,305,294$, and $283 \mathrm{deg}$ azimuth; or 141, 152, $163,174.5,185.5,197,208$, and $219 \mathrm{deg}$ azimuth. In one condition, the speakers were placed in a vertical orientation, with the span centered symmetrically about 0 deg elevation, that is, with speakers positioned approximately at $\pm 5.5, \pm 17, \pm 28$, and \pm 39 deg elevation. Each subject was tested in one of the eight conditions produced by the factorial arrangement of these four orientation conditions and the availability of vision to the subject.

Vision was controlled by having subjects in the no-vision condition wear plastic swim goggles that had been painted black. The goggles had a rubber pad around the eye that provided a reasonably good seal to the head, and no subject reported being aware of anything but a formless fringe of light around the edges of the goggles. The subjects wore the goggles during the previews, practice, and testing, but they were removed between the practice and test session. The subjects in all conditions were allowed to see the entire speaker array before the practice and test sessions. An equal number of subjects (10) was tested in each condition.

The subjects were provided with two previews of each speaker and given 16 practice trials before the 40 test trials began. No feedback was provided. Each speaker was presented equally of ten and in a random order in both practice and testing, with the restriction that no speaker be presented twice in succession.

\section{Results}

The effects of vision in the four conditions were assessed separately by submitting the data for each span orientation to ANOVA, with the eight speaker positions as a within-subjects factor and vision as a between-subjects variable. The dependent measure in all cases was the average error, defined as the mean absolute angular difference between the presented and reported speaker positions.

The only significant effect in the analysis of average error for the horizontal span centered in front of the subjects was the influence of vision $[\mathrm{F}(1,18)=16.47]$. The average-error scores associated with each speaker for the vision and no-vision condition are plotted in Figure 1. The mean average error, collapsed across the eight speakers, was $1.20 \mathrm{deg}$ when vision was available to the subjects and $3.98 \mathrm{deg}$ when it was not.

The ANOVA of the average-error scores in the span oriented to the back of the head (Figure 2) revealed that both the position of the speakers within the array $[F(7,126)=6.56]$ and the interaction of vision and spatial position $[\mathrm{F}(7,126)=2.20]$ were significant. The position effect was due to the general tendency for average error to be lower near the middle of the array, and the effect was slightly more pronounced when vision was available. Overall, there was no difference in average error observed in the array behind the head when vision was provided (mean $=5.71)$ and when it was not $($ mean $=5.46)$.

In the case in which the array was positioned along the left side of the head (Figure 3), the interaction 


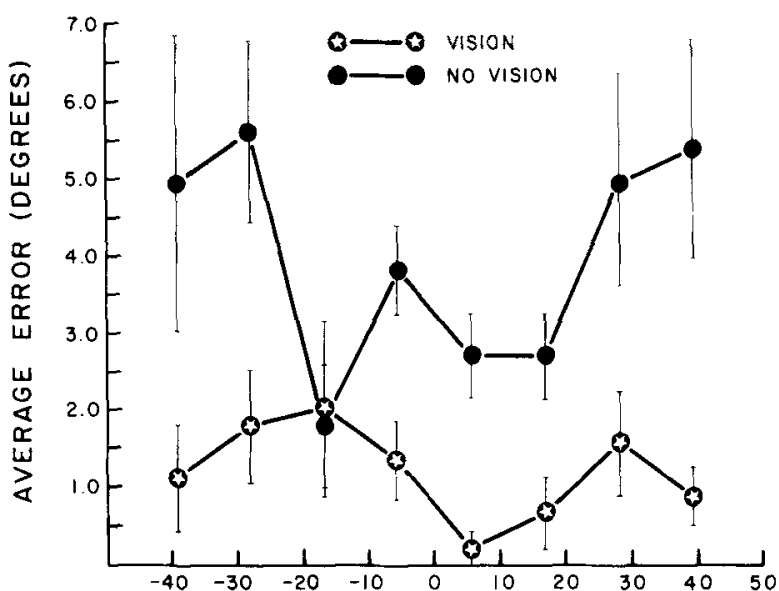

SPEAKER POSITION (DEGREES AZIMUTH)

Figure 1. The mean average error at each speaker position, with and without vision, observed in the front condition. The data are plotted as a function of the speaker position, and the error bars represent the standard error of measurement associated with each point.

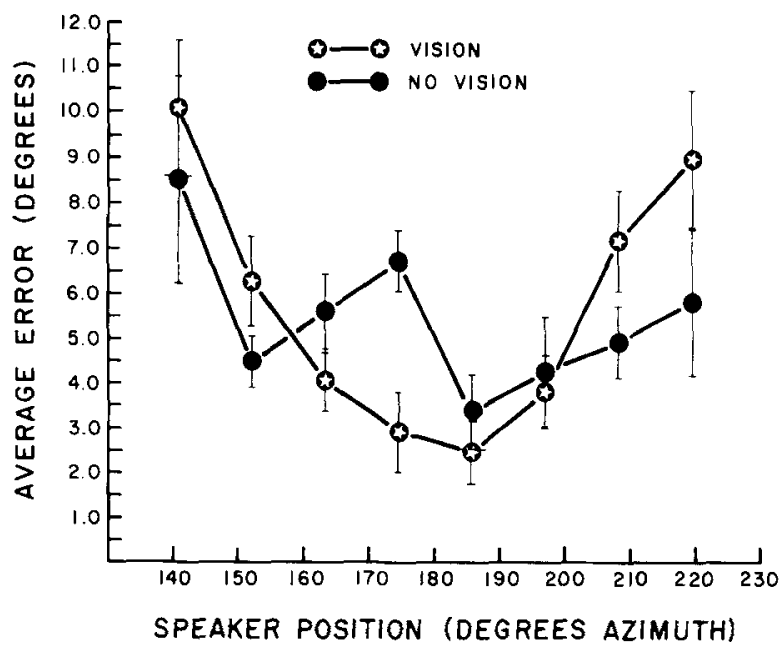

Figure 2. The mean average error at each speaker position. with and without vision, observed in the back condition. The data are plotted as a function of the speaker position, and the error bars represent the standard error of measurement associated with each point.

between vision and the positions in the array was also significant $[\mathrm{F}(7,126)=2.22]$, as was the main effect of vision $[F(1,18)=36.86]$. Speaker position by itself was not a significant factor in the analysis. Vision plays a large role in the determination of average error in the front half of the array, but has little influence with the backmost speakers of the span. At the largest deviation, the difference between vision and no vision was $8.74 \mathrm{deg}$, whereas the difference was only $.35 \mathrm{deg}$ on the next-to-the-backmost speaker. Interestingly, the difference between the two vision conditions was considerable on the backmost speaker in the array, even though subjects were unable to see that speaker.
The position of the speaker in the array was the only significant factor in the ANOVA of the errors associated with the vertically oriented span $[F(7,126)$ $=5.51]$. Figure 4 demonstrates that the effect was essentially that average error was elevated in the topmost speakers and reduced gradually for the lower speakers in the array. The effect was similar with and without vision.

A casual comparison across the four spanorientation conditions indicates that the pattern observed in the four cases was quite different, and that the average error was related to the orientation of the span and the visual condition employed. No speaker position was associated with notably good or bad

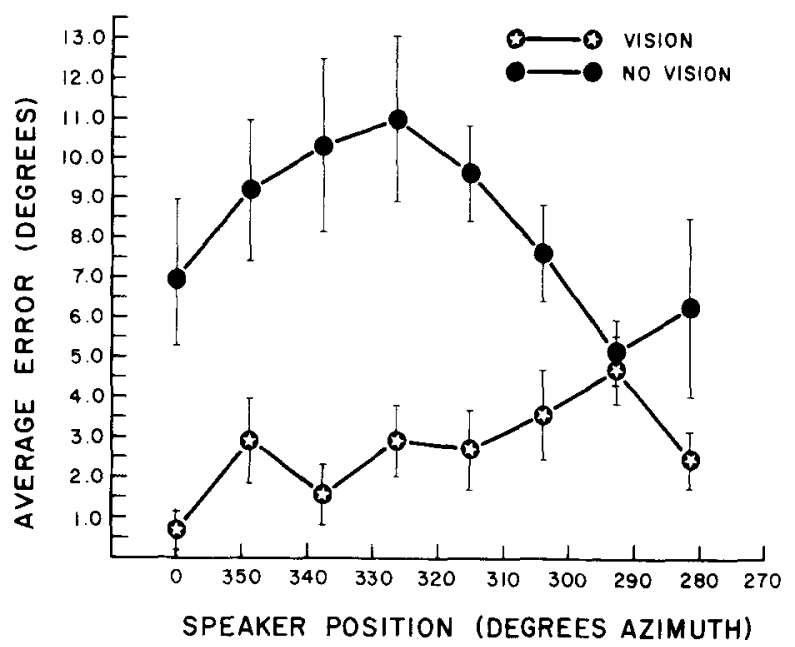

Figure 3. The mean average error at each speaker position, with and without vision, observed in the side condition. The data are plotted as a function of the speaker position, and the error bars represent the standard error of measurement associated with each point.

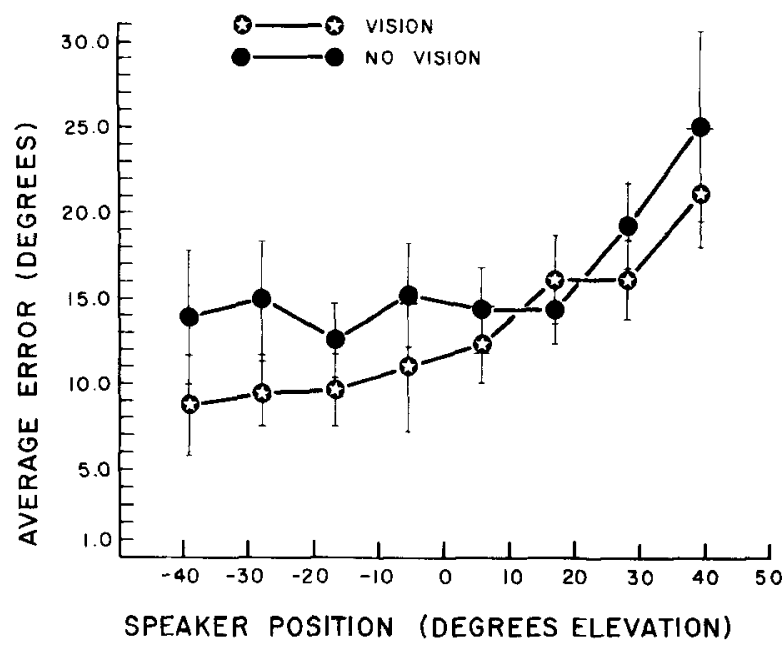

Figure 4. The mean average error at each speaker position, with and without vision, observed in the vertical condition. The data are plotted as a function of the speaker position, and the error bars represent the standard error of measurement associated with each point. 
performance in all conditions, which provides some evidence that the attempts to match the sounds of the speakers used in the experiments were adequate.

\section{Discussion}

Vision had a profound effect on the accuracy of localization judgments in the horizontal plane. The exact effect depended on the particular span orientation under consideration. In the array centered directly in front of the subjects, vision facilitated accuracy with all the speakers, whereas there was an interaction between the speaker position and vision in the other two horizontal array of speakers. However, the interactions observed in these two cases were substantially different. In the case of the span behind the head, the effect was due to greater improvement of performance with vision in the middle speakers of the array. With the array positioned along the left side of the head, the interaction was due to facilitation of judgments of the frontmost speakers with vision.

No difference was observed with and without vision in the vertical plane, which indicates that the effects observed in the horizontal plane were not due to a general disorientation resulting from the restriction of vision or to confusion associated with the response alternatives. To be sure, it would be difficult to account for the three distinct patterns of effect that were observed in the horizontal plane with such a hypothesis, but the lack of a visual influence in the vertical plane eliminates such an interpretation.

It is difficult to understand how there could be a visual influence on judgments made with respect to speakers behind the head, but it is fairly clear that the effect must be related to a generalized frame of reference or perceptual anchor. To be sure, the pattern of response observed behind the head seems primarily related to relatively high accuracy near the center of the array. A visual frame of reference might be expected to effectively define the plane of the head and could thus cause a local influence directly behind the head.

In the two frontal arrays, however, there is evidence of the importance of the direct visualization of the alternatives to produce facilitation. In the span positioned to the side of the head, the interaction indicated that the influence of vision decreased with increasing eccentricity. The observation indicates that in the absolute-identification paradigm, at least some of the high acuity associated with the frontal region is due to the influence of vision. The subjects actually made fewer errors in localizing the backmost speaker when vision was not available than they made when the speaker was directly in front of them. The suggestion here is that, under normal circumstances, the subjects are in some way relying on vision to maintain accuracy in the frontal region.
There is some evidence with the side span for an influence of vision on speakers outside the visual field. There was less error associated with the leftmost speaker of the array in the case in which vision was provided than when it was not, even though this alternative was positioned at $290 \mathrm{deg}$ azimuth and no subject reported being able to see the speaker.

Taken together, these data indicate that vision has a powerful effect on the accuracy of localization judgments in the horizontal plane. There is an indication that there are two distinct influences of vision on sound localization, a specific effect associated with the direct visualization of sources, and a more global effect that can influence performance outside the visual field.

\section{EXPERIMENT 2}

Visual influences on the localization of sounds outside the visual field cannot involve accurate targetdirected eye movements, a factor Jones has argued to be requisite to the visual facilitation of spatial memory (Jones, 1975a; Jones \& Kabanoff, 1975). Thus, the observed visual facilitation of unseen sources is most easily explained as a frame-ofreference effect. At the same time, visible sound sources were facilitated more than sources outside the field of vision. Thus, the outcome of the first experiment provides support for both hypotheses of visual facilitation of auditory localization.

The same is true of separate experiments that have been reported in the literature, but there are important procedural differences across studies that may account for the divergent results. In the absoluteidentification experiments reported by Warren (1970), the auditory stimuli were repetitive clicks presented until the subject completed a response, up to an interval of $4 \mathrm{sec}$. Both Jones (1975b) and Jones and Kabanoff (1975), on the other hand, presented pure tones with step onsets for either 10 or $700 \mathrm{msec}$. Furthermore, Warren used a same-different procedure, whereas Jones and Kabanoff employed a singleinterval signal-detection paradigm. In both instances, Warren's experiments contained a minimal memory component in the task, whereas those of Jones could be expected to place considerable memory demands on the subject, if indeed spatial memory does play a role in auditory localization.

The experiments of Jones and Kabanoff were directed primarily at the question of eye movements in visual facilitation. They were quite successful in this regard, but accuracy was not routinely assessed in total darkness to establish a baseline of performance. Likewise, Warren used a restricted number of lighting conditions-namely, total darkness, total illumination, and a single reference light directly 
in front of the subject. Therefore, the data he reports cannot truly differentiate between a frame of reference and a local influence of vision.

These specific points are not raised as fundamental objections to the validity of these measurements, but rather to illustrate that these experiments were designed to demonstrate a specific effect. As such, the outcomes do not constitute contradictory evidence, but indicate that, under the proper conditions, both the frame-of-reference and the memory-facilitation effects attributed to vision can be demonstrated to improve localization.

The present experiment represents an attempt to manipulate these two visual effects selectively and to investigate some of the parameters in their determination. The basic strategy involved the reduction and control of both the visual and acoustic environments to which subjects were exposed and the manipulation of the correspondence between the spatial information in the two modalities. Differences in the procedures of experiments that have been reported as support for the frame-of-reference and memorystabilization hypotheses of visual facilitation indicate that there may be differences between the two effects in the optimum spatial and temporal relationship between visual and auditory stimuli. Therefore, given that these two effects represent independent processes, there should be an interaction between the timing and placement of visual markers with respect to sound-source positions in the determination of the accuracy of localization judgments.

It takes about $200 \mathrm{msec}$ to orient the eye (Yarbus, 1967), so facilitation should be expected only for visual stimuli greater than $200 \mathrm{msec}$ in duration. Furthermore, a visual stimulus that only precedes a brief auditory stimulus in time should be expected to provide a frame of reference for auditory judgments but should have little influence on spatial memory. Likewise, a visual stimulus that stays on after a brief sound should facilitate spatial memory but cannot provide a frame of reference for a sound that has already terminated. Of course, the neural representations of a stimulus do not follow the exact time course of the stimulus itself, but these generalizations must be true to a close approximation if the concepts of frame of reference and memory facilitation are meaningful. The following experiments used this logic in an attempt to differentially control the two forms of visual facilitation.

\section{Method and Procedure}

Fifty-five male undergraduate volunteers with apparently normal hearing localized single 200 -msec bursts of white noise presented at $62 \mathrm{~dB}$ SPL. Two TDH-39 matched speakers were fixed on the $2.25-\mathrm{m}$ hoop at $\pm 1.0 \mathrm{deg}$ azimuth. The subject's chair, with headrest and bite plate, was $2.10 \mathrm{~m}$ from the speakers, on the opposite side of the speaker hoop. The subjects were provided with a response box that had two buttons. The ITI was $10 \mathrm{sec}$.
Visual stimuli were provided by six light-emitting diodes (LEDs) set in foam rubber mounted in front of the speakers. The LEDs were arranged in pairs positioned at $\pm .7, \pm 1.0$, and $\pm 1.4 \mathrm{deg}$ azimuth, approximately $1 \mathrm{deg}$ below the plane of the speaker cones. There were 11 visual environments in which performance was assessed. Nine of these were produced by the factorial arrangement of the three spatial positions of the lights and three temporal parameters of light presentation. Specifically, the lights were either 1,000 msec long and synchronous with the sound in either onset or offset, or $200 \mathrm{msec}$ in duration and in total synchrony with the auditory stimulus. Performance was measured in total darkness in two groups, one of which was tested at the start of the experiment, and the other at the end.

The lights were always presented in pairs and provided no discriminative information by themselves. The subjects exposed to LEDs were asked to "watch for the lights on each trial, and use them as a spatial marker if you can," but their response was always made with respect to the sound-source position. Except for the light produced by the LEDs, the room was totally dark. The lights were clearly visible, but did not provide enough illumination to allow any contours in the environment to be seen. An equal number of subjects (five) were tested in each of the 11 conditions.

The subjects were provided with two previews of each speaker and given 16 practice trials before the 40 test trials began. No feedback was provided. Each speaker was presented equally often and in a completely random order.

\section{Results}

The data were scored by calculating the $\mathrm{d}^{\prime}$ and beta score for each subject, and submitting these two sets of data to separate ANOVAs. The design was one involving the factorial arrangement of two threelevel factors, the timing and spacing of the lights, and two baseline measurements, so that the data were not organized in a convenient factorial design. Furthermore, particular questions were of critical importance here, such as the equivalence of the two measurements made in total darkness, so that the analysis was carried out by submitting the dependent measures to 11-factor one-way ANOVAs, and specific comparisons were defined by orthogonal weighting coefficients.

The 10 sets of weights used in the analysis are provided in Table 1. The first two sets concern the two measurements made in total darkness: The first set of weights compares performance in the dark with that observed in all the lighted conditions combined, and the second compares the first and second measurements taken in total darkness. The third and fourth comparisons test the linear and quadratic trends across the three spacings of the $200-\mathrm{msec}$ light presentations, and the fifth tests the overall difference between performance with a 200 -msec and a 1,000 msec light.

The remaining comparisons deal with the differences between the cases in which the light preceded the sound and those in which the light remained on after the sound was turned off. The sixth and seventh set of weights test, respectively, for parallel linear and quadratic trends across light spacings in the two lighting conditions. The eighth comparison, however, tests for linear trends across spacings that are in 
Table 1

The 10 Orthogonal Weighting Coefficients Used to Assess the Effects of the 11 Lighting Conditions

\begin{tabular}{|c|c|c|c|c|c|c|c|c|c|c|c|}
\hline & \multicolumn{11}{|c|}{ Lighting Condition } \\
\hline & \multicolumn{3}{|c|}{ Cotemporal (in Degrees) } & \multicolumn{3}{|c|}{ Preceding (in Degrees) } & \multicolumn{3}{|c|}{ Trailing (in Degrees) } & \multicolumn{2}{|c|}{ Darkness } \\
\hline & \pm .7 & \pm 1.0 & \pm 1.4 & \pm .7 & \pm 1.0 & \pm 1.4 & \pm .7 & \pm 1.0 & \pm 1.4 & First & Second \\
\hline ( 1$)$ & 2 & 2 & 2 & 2 & 2 & 2 & 2 & 2 & 2 & -9 & -9 \\
\hline ( 2) & 0 & 0 & 0 & 0 & 0 & 0 & 0 & 0 & 0 & 1 & -1 \\
\hline ( 3) & 1 & 0 & -1 & 0 & 0 & 0 & 0 & 0 & 0 & 0 & 0 \\
\hline ( 4) & 1 & -2 & 1 & 0 & 0 & 0 & 0 & 0 & 0 & 0 & 0 \\
\hline ( 5) & 2 & 2 & 2 & -1 & -1 & -1 & -1 & -1 & -1 & 0 & 0 \\
\hline (6) & 0 & 0 & 0 & 1 & 0 & -1 & 1 & 0 & -1 & 0 & 0 \\
\hline ( 7) & 0 & 0 & 0 & 1 & -2 & 1 & 1 & -2 & 1 & 0 & 0 \\
\hline ( 8) & 0 & 0 & 0 & 1 & 0 & -1 & -1 & 0 & 1 & 0 & 0 \\
\hline (9) & 0 & 0 & 0 & 1 & -2 & 1 & -1 & 2 & -1 & 0 & 0 \\
\hline (10) & 0 & 0 & 0 & 1 & 1 & 1 & -1 & -1 & -1 & 0 & 0 \\
\hline
\end{tabular}

opposite directions between the two 1,000-msec conditions, and the ninth set tests for the same relationship with quadratic trends. The tenth set of weights defines a direct comparison of the cases in which the lights remained on after the sound was turned off with those in which the light preceded the sound.

The ANOVA of d' scores revealed that there were significant differences across the 11 conditions $[F(10,44)=2.50]$. Comparisons $3[F(1,44)=4.02]$, $5[F(1,44)=4.68]$, and $9[F(1,44)=9.37]$ were significant. The mean $d^{\prime}$ scores are plotted in Figure 5.

The significance of the third comparison was due to the fact that, with totally synchronous lights and sounds, $\mathrm{d}^{\prime}$ was very low with the closest spacing of the lights and improved linearly with increased spacing, such that, with the lights separated by $2.8 \mathrm{deg}$, performance was about the same as it was in total darkness. Clearly, this observation was related to a detrimental effect on performance at the closer spacings. The significance of the fifth comparison also reflects this trend: Overall, $d^{\prime}$ was lower with the 200 -msec lights than it was with the 1,000 -msec visual stimuli.

The significance of Comparison 9 was due to the fact that vision facilitated performance with different spatial positionings of the lights when the lights came on before the sound than was the case when the lights remained on after the sounds went off. With the closest spacing of the lights, performance was best when the lights preceded the sound. When the lights and sound positions were cospatial, on the other hand, the subjects were most accurate if the lights stayed on after the sound was terminated. The comparison clearly indicates an interaction between the temporal and spatial parameters of the lights in the determination of the accuracy of auditory localization.

The ANOVA of beta scores with the same weighting functions revealed that none of the comparisons were significant. The overall beta score was 1.09 , so that the manipulations did not appear to produce any consistent bias in the subjects' responses.

\section{Discussion}

The results indicate that both the spatial and temporal relationships between the lights and sound are effective in influencing the accuracy of auditory localization. Under conditions of total synchrony between the lights and sounds, the effect was detrimental to performance, and only the closest spacing of lights with the preceding lights and the cospatial arrangement with the temporally extended lights produced any improvement over performance in total darkness. Because of the specificity of these effects,

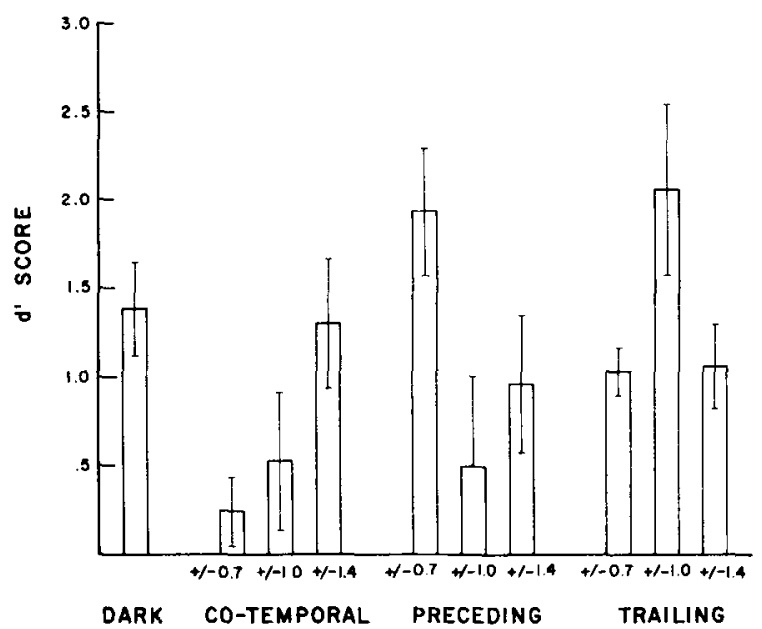

Figure 5. The mean $d^{\prime}$ score associated with each of the $\mathbf{1 0}$ lighting conditions. The cotemporal condition refers to the measurement in which the lights and sounds were both 200 msec and in total synchrony, whereas the preceding and trailing conditions refer to the 1,000 -msec light that was synchronous with the offset and onset of the sound, respectively. The position of the lights in each condition is indicated at $\pm .7, \pm 1.0$, and $\pm 1.4 \mathrm{deg}$ azimuth in each temporal condition of the lights. The speakers were positioned at \pm 1.0 deg azimuth. The darkness condition represents the mean of all 10 measurements made without the benefit of lights. The error bars represent the standard error of measurement associated with each point. 
the overall comparison of performance with and without the benefit of lights was nonsignificant.

The performance with 200 -msec lights indicates that, at least for unpracticed observers, visual information was incorporated into the judgments of sound position, even though the effect was to degrade accuracy. This indicates that, in the normal situation, vision plays a large role in the perception of space and subjects find it difficult to ignore visual information. This interpretation is in accordance with a similar finding in adaptation to a discordance between visual and auditory direction, in which observers find it difficult not to attend to visual stimuli, even though they are instructed to do so (Canon, 1970). It seems likely that, with extended training, subjects could learn to disregard the lights, but the immediate effect observed here was that the lights would degrade performance.

It is not clear why the deleterious effects of these 200-msec lights should decrease as the spacing between the lights was increased. It is possible, however, that the subjects found the more widely spaced lights easier to ignore or that there were some visual localization problems that decreased with the increased spacing of lights across conditions. The fact that there were detrimental effects at all, on the other hand, may be attributable to the fact that, according to the measurements of Yarbus (1967), the physical orientation of the eye to a visual stimulus takes approximately $200 \mathrm{msec}$ to complete. Therefore, the completion of visual orientation and the termination of the light should have been relatively close in time. It is likely, therefore, that the short duration of the lights, and not the synchrony with the sounds, produced the observed effects. An interesting empirical question would be: What temporal duration of the lights and sound would be sufficient to produce facilitation relative to performance in total darkness?

The observations relating facilitatory effects to the spatial positions of the 1,000 -msec lights and the temporal relation to the sound indicated that there are two distinct components to such facilitation. When the lights came on before the sound, performance was best with the most closely placed lights, but, when the lights remained on after the sound went off, performance was best with spatially coincident lights and sound.

The dual nature of the effect and the two processes discussed in the literature seem to correspond. A general frame of reference, to be optimally useful, should be available before specific stimuli are presented, so that the case in which the lights came on before the sound might well reflect this process. If this is true, it is reasonable that the optimum stimulus should be one that orients the subject to a position between the two alternatives. The most closely spaced lights, assuming subjects orient to one or the other of the lights, is the only instance in which this would be the case. From this interpretation, it would have to be admitted that a single light source halfway between the alternatives would have been effective in producing an effect. It is worthwhile to note that Warren (1970) has already shown that this stimulus arrangement does provide an adequate frame of reference for the facilitation of localization judgments.

Visual facilitation of spatial memory is a possible explanation of the influence of the lights that remained on after the sound was terminated. The facilitation was due to an influence in time after the discriminative stimulus, so the process must, in some sense, involve memory. Furthermore, the fact that spatial correspondence was required to produce any measure of facilitation is in accordance with the suggestion that target-directed eye movements are required to produce visual facilitation of position memory (Jones, 1975a; Jones \& Kabanoff, 1975).

The results constitute evidence for a complementary relation between the frame-of-reference and the memory-stabilization hypotheses of visual facilitation. Those defending the respective points of view have used the reasonable strategy of employing paradigms that maximize the effect of interest. In some sense, this is a general strategy of science, but there has to be a balance between the production of a phenomenon under controlled conditions and the study of a phenomenon under limited conditions. In this particular case, it appears that, in order to optimize one aspect of visual facilitation, a second aspect of the effect was minimized, at least in the experiments used as primary support for the two prominent theories. The results reported here indicate that both processes are involved in visual facilitation, each with different governing parameters and, therefore, possibly complementary effects in full-context environments.

\section{REFERENCES}

AnNis, R. C. Auditory and visual interaction in spatial localization tasks. Unpublished master's thesis, Queen's University, 1974.

Aronson, E., \& Rosenbloom, S. Space perception in early infancy: Perception within a common auditory-visual space. Science, 1971, 172, 1161-1163.

Canon, L. K. Intermodality inconsistency of input and directed attention as determinants of the nature of adaptation. Journal of Experimental Psychology, 1970, 84, 141-147.

Fishe R, G. H. Spatial localization by the blind. American Journal of Psychology, 1964, 77, 2-14.

Jack, C. E., \& Thurlow, W. R. Effects of degree of visual association and angle of displacement on the "ventriloquism" effect. Perceptual and Motor Skills, 1973, 37, 967-979.

JACKson, C. V. Visual factors in auditory localization. Quarterly Journal of Experimental Psychology, 1953, 5, 52-65.

JoNes, B. Spatial perception in the blind. British Journal of Psychology, 1975, 66, 461-472. (a)

Jones. B. Visual facilitation of auditory localization in school 
children: A signal detection analysis. Perception \& Psychophysics, 1975, 17, 217-220. (b)

Jonis. B.. \& Kabanorf, B. Eye movements in auditory space perception. Perception \& Psychophysics, 1975, 17, 241-245.

LACKNFR. J. R. The role of posture in adaptation to visual rearrangement. Neuropsvchologica, 1973, 11, 33-44. (a)

LACKNER, J. R. Visual rearrangement affects auditory localization. Neuropsychologica, 1973, 11, 29-32. (b)

Mclaughion, S. C., \& Bower, J. L. Auditory localization and judgements of straight ahead during adaptation to prism. Psuchonomic Science, 1965, 2, 283-284.

Muir, D., \& FiELD, J. Newborn infants orient to sounds. Child Development, 1979, 50, 431-436.

Radeau, M., \& Bertelson, P. The after-effects of ventriloquism. Quarterly Journal of Experimental Psychology, 1974, 26, 63-71.

SHERRINGTON, C. S. The integrative action of the nervous system. Cambridge, England: Cambridge University Press, 1947.

Spelke. E. Infants' intermodal perception of events. Cognitive Psychology, 1976, 8, 553-560.

Thurlow. W. R., \& JACK, C. E. Certain determinants of the "ventriloquism effect." Perceptual and Motor Skills, 1973, 36, 1171-1184. (a)

Thurlow, W. R., \& J Ack, C. E. Some determinants of localization-adaptation effects for successive auditory stimuli. Journal of the Acoustical Society of America, 1973, 53, 1573-1577. (b)

Thurlow, W. R., \& KerR, T. P. Effect of a moving visual environment on localization of sound. American Journal of Psychology, 1970, 83, 112-118.

Wallach, $H$. The role of head movements and vestibular and visual cues in sound localization. Journal of Experimental Psychology, 1940, 27, 339-368.

WARREN, D. H. Intermodal interactions in spatial localization. Cognitive Psychologv, 1970, 1, 114-133.

Wertheimer, M. Psychomotor coordination of auditory and visual space at birth. Science, 1961, 134, 1692.

Yarbus, A. L. Eye movements and vision. New York: Plenum Press, 1967.

(Received for publication April 10, 1980; revision accepted September 3,1980.) 